\title{
Inventory of Epidemiological Data Sources in Preparation of a Joint Analysis of Zoonotic Disease Information
}

\author{
Anna Wendt*1, Lothar Kreienbrock ${ }^{1,2}$ and Amely Campe ${ }^{1,2}$ \\ ${ }^{1}$ Department of Biometry, Epidemiology and Information Processing, University of Veterinary Medicine, Hannover, Germany; ${ }^{2} \mathrm{WHO}$ \\ Collaborating Centre for Research and Training in Veterinary Public Health, Hannover, Germany
}

\section{Objective}

An inventory of epidemiological data sources in Germany is being conducted to evaluate a possible integration of zoonotic disease information.

\section{Introduction}

For the surveillance of zoonoses a vast amount of digital data is routinely being collected on three sectors, humans, animals and food. Depending on the origin and the purpose of the data collection, this information is stored in different databases. Interfaces between these data sources are rare. Against the background of One Health and regarding the high costs of collecting new data, there are growing demands to make use of existing data to improve the surveillance of zoonoses. It is particularly required to combine data from different sectors to improve early warning and prompt detection of zoonotic disease events.

It is hypothesized that a joint use of information is regarded to be more productive than the traditional segregated sector approach. However, prior to evaluating this, it has to be determined if the existing data are suitable for a joint analysis. Therefore the following questions have to be answered:

- Do the data fit the purpose and cover the required information (content, population of interest, detail, time, space) in an adequate quality (i.e. timeliness, accuracy)?

- Can they be combined, if they originate from different sources?

A systematic description and documentation of the existing datasources and their content is necessary to evaluate the feasibility. Until now, in Germany no inventory exists of those datasources, in which data on zoonoses are routinely collected. In addition to the challenges of a uniform description of datasources from different sectors (human/ animal/food), there are difficulties that come along with the federal structures of data collection. In Germany, if not mandatory, data on infectious disease events are not collected nationwide but on a county level. Hence, they are neither consistent, nor is there a one-stop shop for access.

\section{Methods}

A questionnaire with 45 variables was developed to enable a factual description of different datasources which routinely collect data on zoonoses. Informations on content, format, transparency for secondary use and some quality indicators of the datasources are collected in a standardized manner to enable a subsequent estimation of their suitability for a specific analysis.

\section{Results}

Up to now, 12 datasources were identified as relevant in Germany. These heterogeneous datasources were described using the developed questionnaire in personal interviews.

First results show that there are no routines established for a systematic and rapid exchange of information between the different sectors. A current exchange depends on the motivation of the individuals involved.

An evaluation of the suitability of existing datasources depends heavily on the purpose and cannot be conducted before formulating precise hypothesis.

One of the most frequently demanded hypotheses is to improve the early detection. For this, the yet existing data are not suitable, as they mostly lack timeliness. Whereas data on notifiable animal or human disease outbreaks have to be reported within a few days to national authorities, informations on zoonoses, that are not classified as notifiable pathogens, are being published months later. More current information on not notifiable pathogens is divided among more than one information system. For other purposes the use of a cross-sectoral information integration still has to be evaluated.

\section{Conclusions}

The developed questionnaire facilitates an overview of existing information. It captures what information is currently available, in what form and extent and where it can be received. Especially for not centralized data holdings a systematic description of the existing information is required in order to assess their usefulness for subsequent analyses. Depending on the type of analysis, further information on the dataset might be required. As the secondary user has not been involved in the primary data collection process, the required information should be easily available in form of suitable metadata descriptions or through a close cooperation with the data owner.

\section{Keywords}

zoonotic disease surveillance; secondary data; metadata description

\section{Acknowledgments}

This work is supported by TMF - Technology, Methods, and Infrastructure for Networked Medical Research (V080-01)

*Anna Wendt
E-mail: anna-maria.wendt@tiho-hannover.de 